\title{
First-line all-oral NORCAP (vinorelbine/capecitabine) might be alternative to taxane-based chemotherapy for HER2-negative metastatic breast cancer
}

\author{
Kadri Altundag ${ }^{1}$
}

Received: 21 October 2016/Accepted: 10 November 2016/Published online: 17 November 2016

(C) Springer Science+Business Media New York 2016

To the Editor,

I read with great interest the article by Cazzaniga et al. [1] in which they investigated metronomic chemotherapy with oral vinorelbine (mVNR) and capecitabine (mCAPE) (VICTOR-2 study) as a first- or second-line treatment in advanced HER2-negative breast cancer patients. The primary endpoint was the clinical benefit rate (CBR); secondary endpoints were toxicity, objective response rate (ORR), and progression-free survival (PFS).Eighty patients were evaluable for the primary efficacy analysis. Median age was 65.3 years; most patients had HR-positive tumors (65\%). The CBR was 45.7 and $51.1 \%$ in first- and $\geq$ secondline therapy, respectively. The ORR was $35.5 \%$ in first line and $25.6 \%$ in $\geq$ second line. The median duration of response was 11.3 and 6.4 months and PFS rates at 1 year were 24.3 and $22.2 \%$, respectively. The authorst noted that the VICTOR-2 study is the first multicenter prospective trial testing the fully oral metronomic combination of VNR and CAPE in a population of advanced HER2-negative breast cancer patients with prespecified analyses of efficacy and safety according to biological subtype, line of treatment, and site of metastatic.disease. However, the recent phase II NorCap-CA223 trial [2] compared the first-line all-oral NORCAP (VNR and CAPE) versus two different taxane-based chemotherapy gemcitabine/paclitaxel
(GEMPAC) or gemcitabine/docetaxel (GEMDOX) for HER2-negative metastatic breast cancer. Median age was 56-58. The CBR was 73\% with NORCAP (36 of 49 patients), 78\% (with GEMPAC (39 of 50 patients), and $80 \%$ with GEMDOC (40 of 50 patients). ORR were $33 \%$ (16 of 49 patients), 24\% (12 of 50 patients), and 50\% (25 of 50 patients), respectively; median PFS was 7.6, 9.0, and 11.4 months, respectively. Median overall survival was 30-31 months with all regimens. In conclusion, all-oral NORCAP is an active first-line chemotherapy regimen and might be offered as an alternative to first-line taxane-based therapy for HER2-negative metastatic breast cancer.

\section{References}

1. Cazzaniga ME, Cortesi L, Ferzi A et al (2016) Metronomic chemotherapy with oral vinorelbine (mVNR) and capecitabine (mCAPE) in advanced HER2-negative breast cancer patients: is it a way to optimize disease control? Final results of the VICTOR-2 study. Breast Cancer Res Treat 160:501-509

2. Cinieri S, Chan A, Altundag $K$ et al. Final results of the randomised phase II NorCap-CA223. Trial comparing first-line alloral versus taxane-based chemotherapy for HER2-negative MBC. Clin Breast Cancer. doi:10.1016/j.clbc.2016.06.014

This letter to the editor refers to the article available at doi:10.1007/ s10549-016-4009-3 and a rebuttal letter to this letter to the editor is available at doi:10.1007/s10549-016-4056-9.

Kadri Altundag

altundag66@yahoo.com

1 Tria Residence, A Block No: 8, Yildizevler Mah. Cankaya, Ankara, Turkey 